\title{
Fabrication of Solid Models by Sequential Solidification of Liquid Polymer
}

\author{
Yoji MARUTANI and Takayuki KAMITANI \\ Faculty of Engineering, Osaka Sangyo University, \\ 3-1-1 Nakagaito, Daito, Osaka 574, Japan
}

\begin{abstract}
The new manufacturing technology called the rapid prototyping (RP) or layered manufacturing can fabricate very complicated solid objects fully automatically. RP industry is now growing quickly and new additive methods of manufacturing are coming along. Among many types of RP which have been put to practical use by this time, the system using liquid photopolymer and a laser beam is called "photo-shaping" or "stereolithography", and nowadays most popular. Here, the principle of it is first described. Then the newly proposed methods using liquid polymer are introduced. These aim to lower the cost or to enhance the ability of the systems. Finally characteristics of the resin necessary in the application of the solid models are pointed out.

Keywords: rapid prototyping, solid model, photopolymer, CAD, mold
\end{abstract}

\section{Introduction}

The rapid prototyping $(\mathrm{RP})$ using an ultra-violet (UV) laser and photopolymer are usually called photo-lithography (in USA) or photo-shaping (in Japan)[1]. It was developed about 10 years ago as a new manufacturing method of making mechanical parts directly from digital data such as CAD data. RP is now used in industry for the verification of CAD design and the functional checking of machine parts at the development stage of new products. After the sucoess of the photo-shaping, various types of RP, some of which are suitable for metal or ceramics process, have been developed, thus broadening the application fields.

At present the leading technology of RP is still photo-shaping. As the application of the solid models extend to many fields, the needs for the resin has become more severe and diversified. Acoordingly current situation and future trend of photo-shaping are discussed here, touching upon the resin because it is the key element of the method.

\section{Types of the photo-shaping}

2.1 The system using UV laser and photopolymer

The configuration of the photo-shaping is divided into two clases by layer lamination process. Fig.1 shows the principle of "free surface method"[2]. At first, a layer is solidified by scanning UV laser beam on the surface of the liquid photopolymer according to the lowest sectional pattem of the CAD data. The irradiated area is selectively solidified to form thin layer having the sectional pattern. Then the liquid layer is formed on it and irradiated again to form the next sectional pattern. Thus the layers are stacked each other automatically. These process are continued until the model is completely shaped. Fig.2 shows the full configuration of the system. The laser beam is scanned by two moving mirmors. By systems on the market, the maximum size of the model is $800^{*} 1000^{*} 500 \mathrm{~mm}$, and the maximum scanning speed is $20 \mathrm{~m} / \mathrm{sec}$, which depends upon the photosensitivity of the resin.

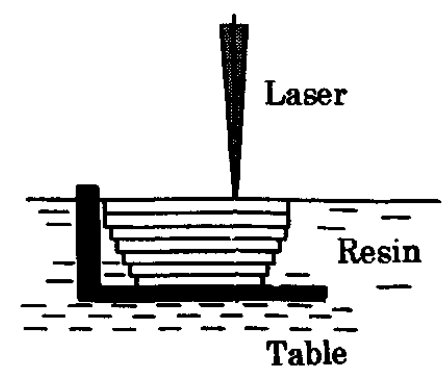

Fig.1 Principle of photo-shaping

Both urethane-acrylate base resins and epoxy base 
resins for UV laser are currently most popular, but characteristics of hardened material do not always satisfy user demands, because the RP system has much restrictions. Though epoxy is rather slow in reaction, it has such merits as low shrinkage, bw distortion and low viscosity. Unethane has good mechanical and thermal characteristics. Anyway both materials are not as good as the popular engineering plastics, $\mathrm{ABS}$.

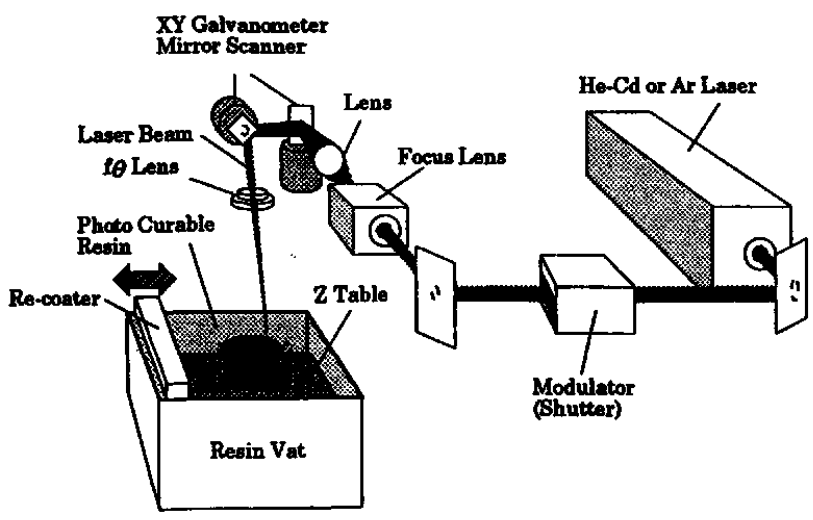

Fig.2 Configuration of photo-shaping machine

The necessary characteristics of the photopolymer for these purposes are:

(1)low visoosity,

(2)long lifetime,

(3)rapid reaction,

(4)good adhesion to solidified layer,

(5)low shrinkage,

(6)mechanical strength,

where (3) and (4) are unique features needed in the photo-shaping. Fig.3 shows an example of the precision of the photo-shaping[3]. The variance of the size is less than $0.1 \mathrm{~mm}$ for the model of $300 \mathrm{~mm}$ square. Fig.4 is a solid model fabricated by this method.

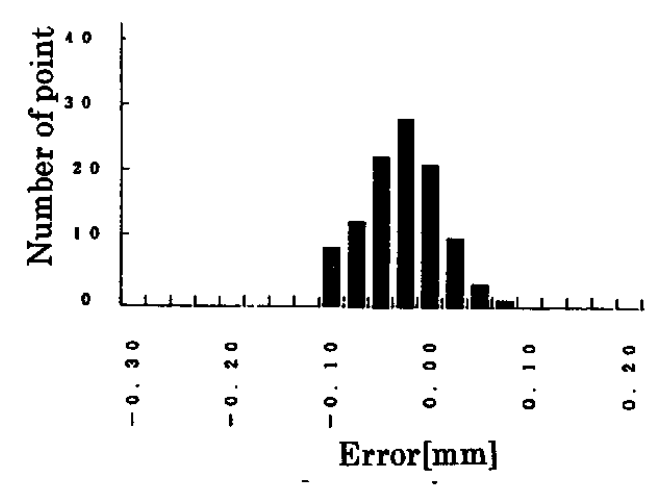

Fig.3 Data showing the precision

2.2 Use of wax for the support[4]

An image of each layer is produced on an electrostatically charged erasable glass plate. The image, serving as a photomask, is pasitioned over a thin layer of liquid photopolymer. The resin is then

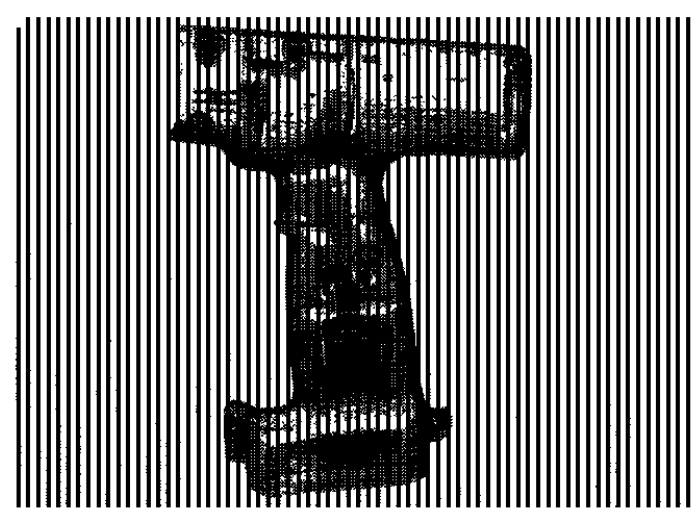

Fig.4 Models fabricated by usual photo-shaping

selectively solidified by a powerful UV light through the mask, creating that layer of the model. After the layer is cured, all unsolidified resin is collected, leaving the hardened areas intact. Melted wax is then spread into the newly created cavities. The wax is cooled and hardened to provide continuous, solid support for the model as it is fabricated. The layer is milled to a smooth, accurate, even surface. A new layer of photopolymer is spread on the milled surface, a new mask is generated on the cleaned glass plate, and the process is repeated until the build is completed. This process needs no support for unstable parts of the model, however needs the cleaning process of the wax.

\subsection{Combination of UV and visible light[5]}

As shown in Fig.5, the resin is highly transparent to the visible light. To obtain thin solid layers, the liquid layer must be originally thin as it is expanded on the solidified surface. The system uses "controlled

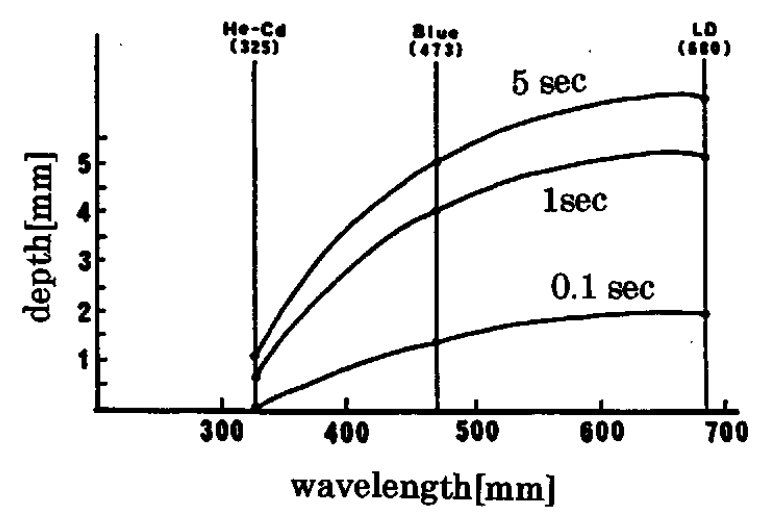

Fig.5 Wavelength and thickness of solidification

surface method" to realize the thin layer, and the layer is formed on the surface of a glass plate. The least thickness of it is $0.1 \mathrm{~mm}$. The method 
combining a visible laser with a UV laser make unprecious part with the visible light as shown in Fig.6. The visible light solidifies the resin very thickly and make total lamination time shorter.

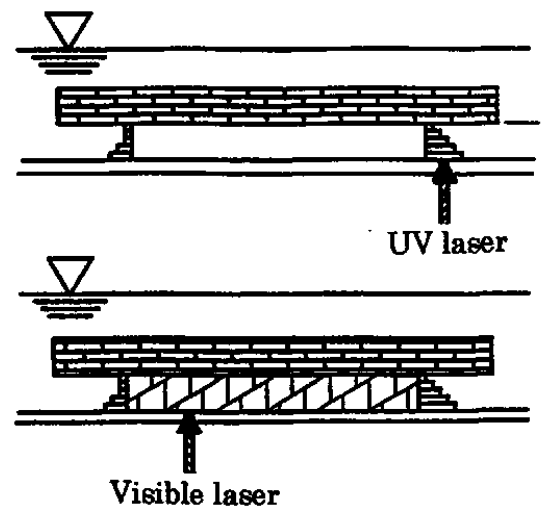

Fig.6 Combination of Visible and UV laser

\subsection{Mixing ceramic powder[6]}

Properties of ceramics are, for example, hardness, heat-resistance and chemical stability. A major problem is that ceramics are too brittle to machine. So the layer additive process is more preferable than the subtractive technology like milling. The usage of a doctor blade process or excluding of a thin film seems difficult because of the high viscosity, and rolling and pressing are chosen to manufacture the slice.

A composite has been produced by mixing ceramic powder with a photopolymer resin. The produced ceramic-binder films are laminated and selectively cured in a RP machine to make "green bodies" . After cleaning the unsolidified resin, the green bodies are fired to burn out the resin and afterwards sintered to achieve full strength. The compasite with $60 \mathrm{vol} \%$ alumina and 40 vol.\% epoxy (HS-660, Asahi Denka Co.) was found suitable.

Some venders use mixed polymer with fillers. These polymers are highly heat-resistive, easy to machining and adhesive.

\section{New method to simplify finishing}

Usually supports or ribs are added to the original $\mathrm{CAD}$ data to prevent parts unconnected to the base plate from floating during shaping process. These also prevent the distortion of the models. Rejecting and finishing the supports are one of the serious problems of the photo-shaping. Here two methods to solve this are mentioned.

\subsection{Supporting by refrigeration[7]}

After expanding the liquid resin, the layer is refrigerated to be solid state. Then it is solidified by laser scanning. When all layers are fabricated, the whole block is heated and the solid model is taken out from the melted resin. To realize this method, a new photopolymer was developed. Temperature at dispense is $95{ }^{\circ} \mathrm{C}$ and cooling temperature is $-20^{\circ} \mathrm{C}$. In the experiments, thickness of the layer: $0.15 \mathrm{~mm}$, laser exposure: $900 \mathrm{~mJ} / \mathrm{cm}^{2}$, laser power. $14.5 \mathrm{~mW}$, scanning speed: $17 \mathrm{~mm} / \mathrm{sec}$.

\subsection{Non-laminating method[8]}

As stacking of the solidified layer is essential in $R P$, the solid model always has steps on its surface. The polishing of the surface is also another problems of $R P$. If the resin is exposed in the liquid in 3-dimension, the layer lamination is unnecessary and the solid models are step-free. One method is to insert a $n$ optical fiber into the resin. But according to our experiment, the solidified part sticks firmly to the end of the fiber and is destroyed with the movement of the fiber. We realized this by using a bubble. As shown in Fig.7, an air bubble is generated at the end of a pipe under the surface of the resin. An UV laser beam is introduced into the resin and solidifies the resin at the surface of the bubble.

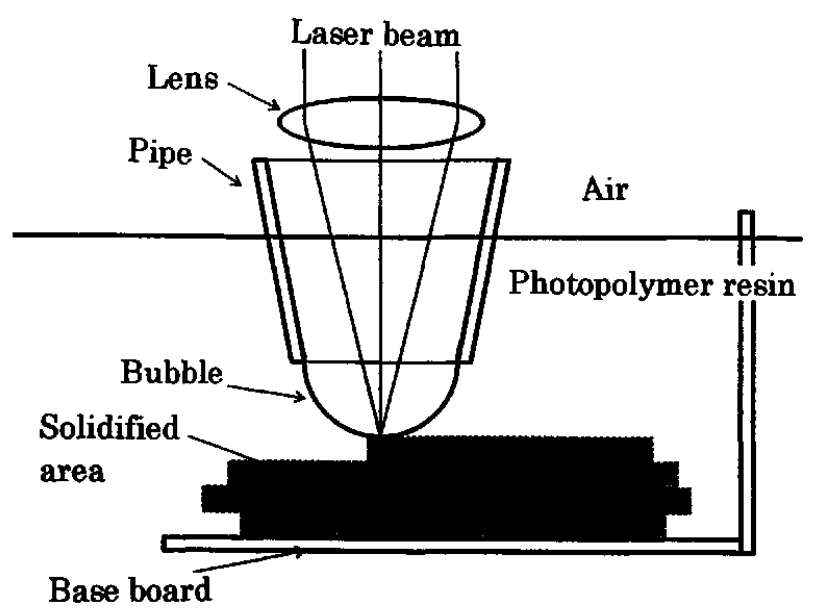

Fig.7 Principle using air bubble

The trajectory of the path of the bubble and beam is solidified 3-dimensionally. The exposure root is not limited so long as it does not interfere with the formarlly solidified area. One of the limitation of this method comes from the deformation of the bubble. As the pressure around the bubble have an effect on the size of the bubble, the inner pressure must be adjusted in proportion to the depth of the bubble. On scanning the exposure spot, the dynamic pressure of the resin deforms the shape of the bubble, and in the worst case, pull it off the pipe. This limits the maximum speed of scanning.

In the experiments, the size of the bubble was $\mathrm{R}=4 \mathrm{~mm}, \mathrm{c}=3 \mathrm{~mm}$. The output of the $\mathrm{He}-\mathrm{Cd}$ laser with 
wavelength $325 \mathrm{~nm}$ was $3 \mathrm{~mW}$ and the focal length of the lens was $50 \mathrm{~mm}$. The resins were TSR20, TSR21 and TSR46(Teijin Seiki Co.) and their visoosities were 1950,440 and 1700 cps respectively. It is well known that the photosensitivity of the radical type resin is lowered by the oxygen in the air. The experimental results shown in Fig.8 shows that this phenomenon is avoided by the under-surface exposure.

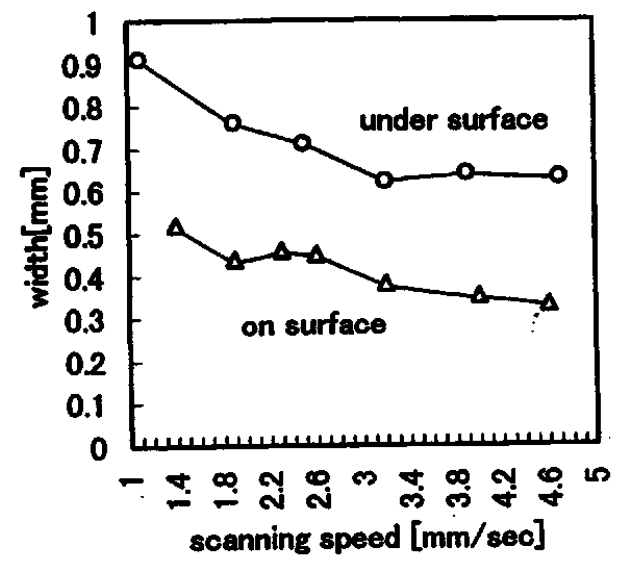

Fig.8 Photosensitivity in the bubble and in the air

The model in Fig.9(a) is made using this method. To make the size of the bubble stable, the exposure was made in the constant depth of $5 \mathrm{~mm}$. Fig.9(b) shows the result of 3 -dimensional scanning. The exposure point was moved in spiral and the spiral shape was made without no steps on its surface.

The requirement for the resin is low visoosity and hardness in solidified state.

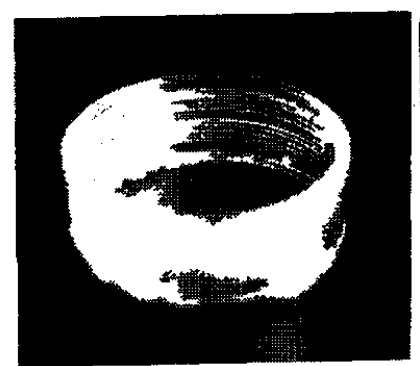

(a)

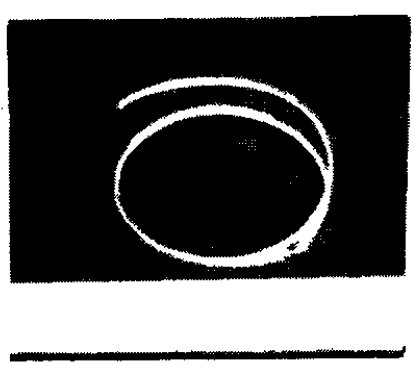

(b)
Fig.9 Models made by the air bubble method

\section{Low cost system}

Present photo-shaping systems have issues in cost and size because of the UV laser assembled in them. As RP becomes more popular, small and inexpensive systems are requested.

\subsection{Use of discharge lamp[9]}

A discharge lamp is used as a UV light source, and to make the fabrication time shorter, multiple spots exposure using optical fibers is employed. The light from the lamp is distributed to these fibers and the output side of each fiber is arrayed linearly and the emitted light are focused on the surface of the photopolymer. The light transmission of each fiber is controlled by the shutter independently according to the pixel data at the corresponding position.

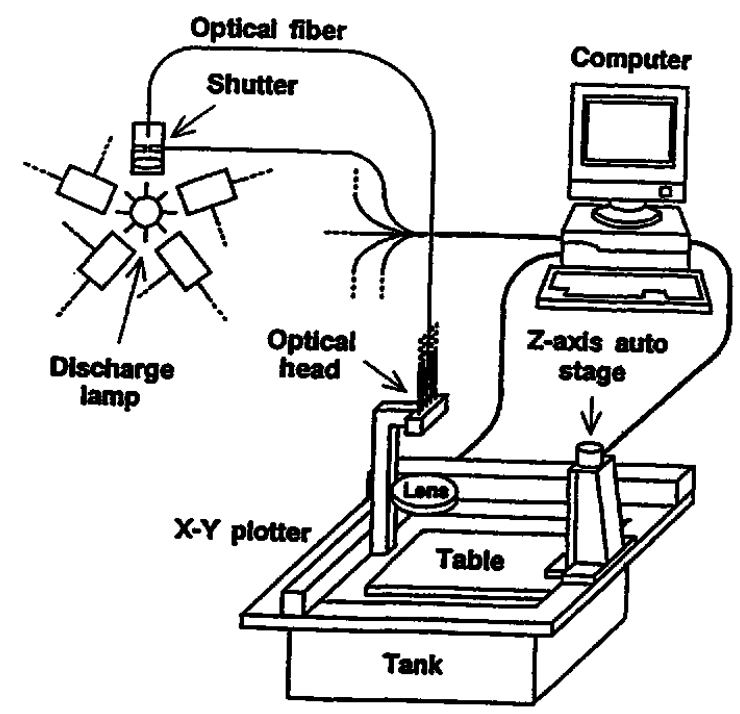

Fig.10 Schematic of the system using discharge lamp

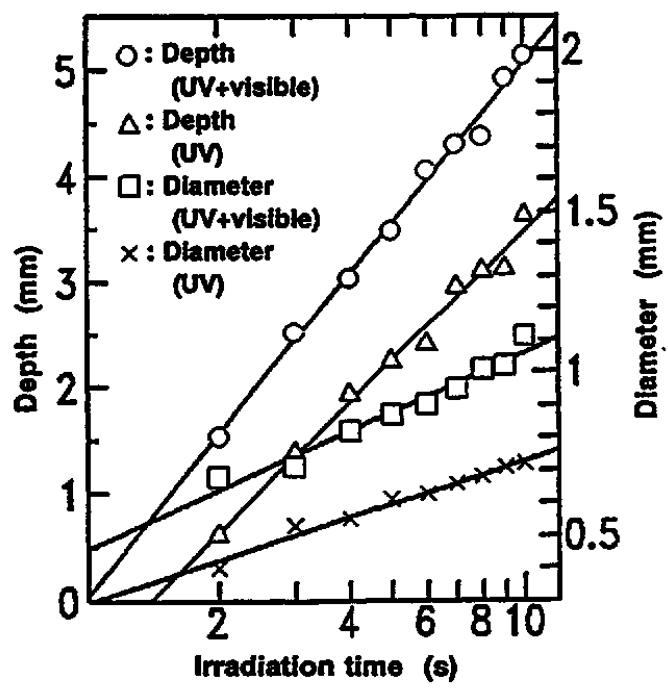

Fig.11 Relationship between irradiation time and size of solidified part

To measure the resolution and solidification rate, the resin(HS660, Asahi Denka Co.) was expased by single fiber. Fig.11 shows the relation between the irradiation time versus the diameter and depth of solidified part. In an example of commencial system using a $100 \mathrm{~mW} \mathrm{UV} \mathrm{laser,} \mathrm{a} \mathrm{layer} \mathrm{of} 0.08 \mathrm{~mm}$ in diameter and $0.2 \mathrm{~mm}$ in thickness is solidified at scanning speed of $770 \mathrm{~mm} / \mathrm{sec}$. The volume production rate in this case is $9.7 \mathrm{~mm}^{3} / \mathrm{sec}$. From Fig.11, our system produces $0.29 \mathrm{~mm}^{3} / \mathrm{sec}$. So about 34 fibers are equivalent to the ability of $100 \mathrm{~mW} \mathrm{UV}$ 
laser.

The requirement for the resin is the broad wavelength sensitivity.

\subsection{Use of thermosetting resin[10]}

Another method to save cost is the combination of thermosetting resin and a infra-red laser. This type of laser is small in size and very economical. In the experimental system, cationic epoxy resin ( thermosetting resin) was irradiated with $\mathrm{OO}_{2}$ laser (maximum output:9.5W, wavelength:10.6 $\mu \mathrm{m}$ ) and solidified layers were stacked to shape solid models. As shown in Fig.12, it was proved that irradiation with high power laser light caused explosive chain thermopolymerization, and the stable operating range for controllable reaction could be enlarged by mixing plastic powder in the resin. A solid model of 20 $\mathrm{mm}$ square in bottom was made by stacking 20 layers (Fig.13).

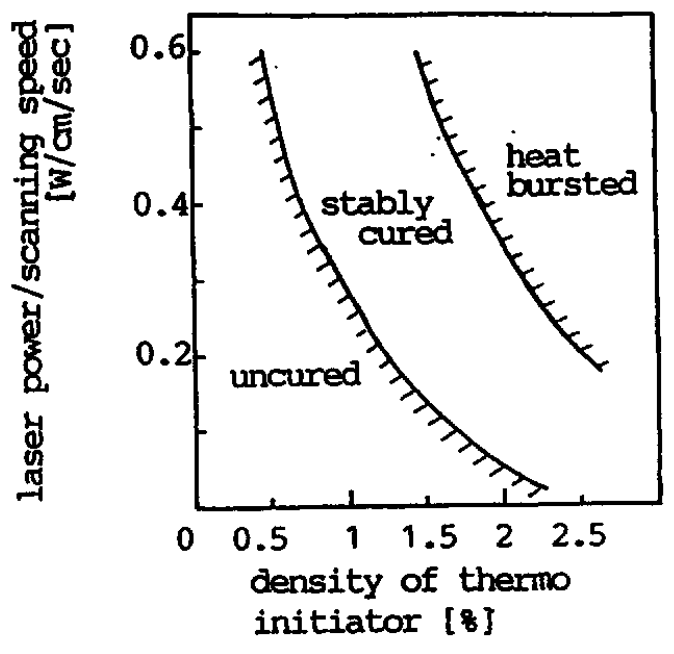

Fig.12 Condition for stable reaction

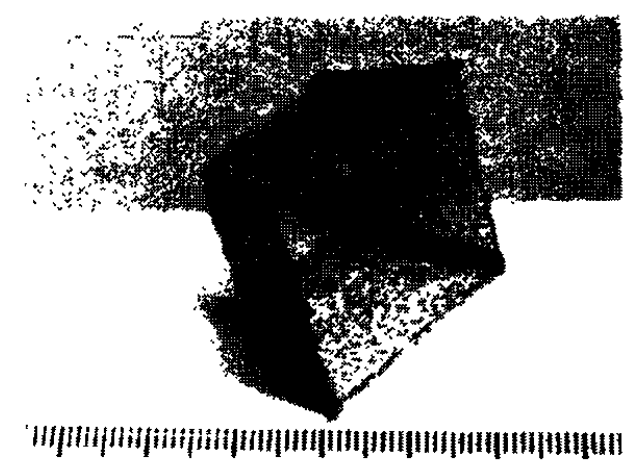

Fig.13 Solid model made by thermosetting resin.

In this method, the most important requirement for the resin is quick chemical reaction.

\section{Application of RP}

Application of RP have penetrated into various commencial branches. Most of the RP models are used for the verification and functional checking of $\mathrm{CAD}$ design. Various new types of RP machine, some of which are suitable for metal and ceramics, have been developed, thus broadening the application field. There are strong needs for the application to die and mold manufacturing, for production of medical parts and micro machine parts in the future.

\subsection{Verification of the design}

To elaborate the outside view of products, solid models must be as soon as possible. The solid models

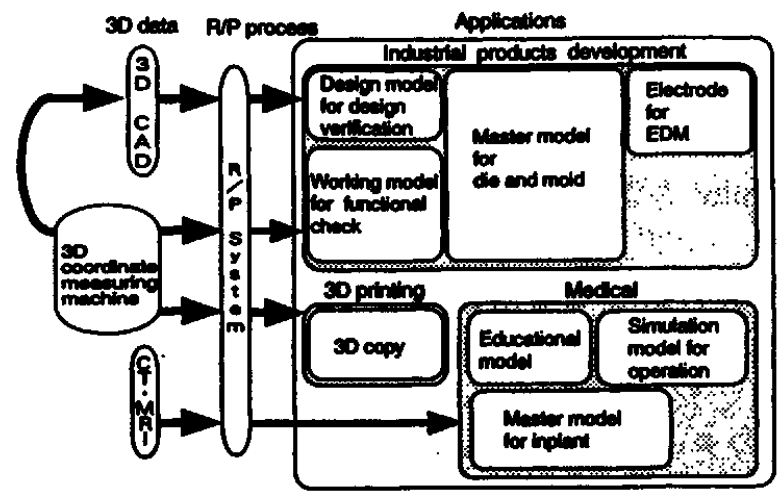

Fig.14 Application of RP[2]

used in this area do not mind the material. So the $\mathrm{RP}$ is suited because complex structure is shaped automatically in a short time. RP is utilized mastly for this purpose.

\subsection{Functional model}

Electronic and mechanical parts are installed to the functional model. So the tenacity of the material, in addition to the accuracy of size, is needed for this purpose. When the resin is too brittle, it is copied to another kind of resin.

\subsection{Making metallic parts by copying}

The main materials for mechanical industry is still metal, and they need metal parts finally. Therefore all kinds of technology have been developed to translate plastic models to metal. The plaster work is adopted for the simple shaped models. When the model is complex, investment casting is selected. In this case the model is covered by ceramic mud and the resin consisting the model is removed by heating to produce the cavity in it. The resin should have low heat expansion, low melting point and easy combustion.

\subsection{Ceramic parts}

To make cramic using the photo-shaped models, 
the rubber model is made first by copying the plastic model. Then ceramic powder with liquid binder is poured into the cavity of the model. After the powder is solidified by the binder, it is taken out from the rubber model and sintered in the furnace.

\subsection{Making die and mold}

One method of making die and mold directly by photo-shaping is to use photopolymer mixed with metal powder [11].

In another method[12] the shape is copied to thermo-setting resin with metal powder. Here the resin consisting of $75 \%$ of alumina and $25 \%$ of epoxy resin. The resin is held at temperature of $150{ }^{\circ} \mathrm{C}$ for $5-6$ hours and solidified thermally. The die made thus is heat-resistive until $250^{\circ} \mathrm{C}$. The model was used for injection mold and more than 20 times of shot were done with shot cycle of $1 \mathrm{~min}$.

Metal dies are made by investment casting as mentionedin 5.3.

\subsection{Medical application}

Models of borns and internal organs of alive human are made using the data from CT or MRI. These are utilized to make a diagnosis or to simulate the surgical operation.

\subsection{Micro machine}

Up to this time the silioon process has been applied for this subject. Therefore the realizable shape is restricted to be flat, because the process is photolithography of the thin wafer. Now the 3-D micro machines can be made freely by RP. Fig. 15 shows a method developed for this purpose[13]. The minimum realizable size is said to be $0.001 \mathrm{~mm}$. Although the micro machine is not clear yet whether it is useful or not.

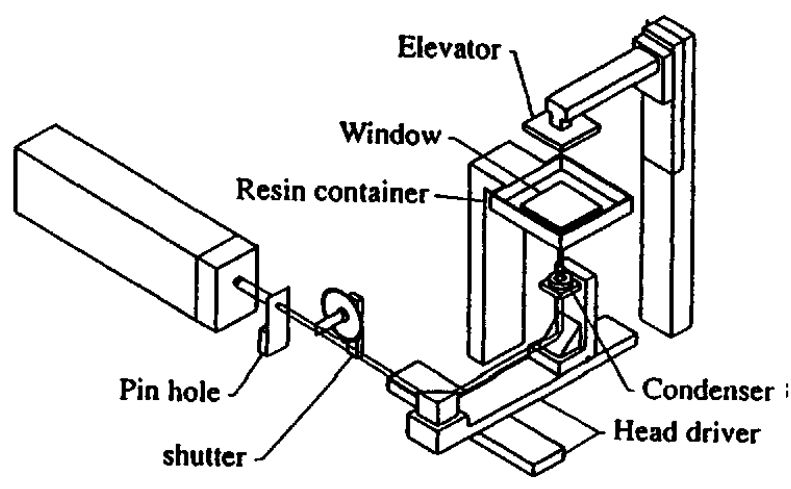

Fig.15 "restricted surface method" for micro machines

\section{Conclusion}

The newly developed manufacturing technologies using liquid resin named "photo-shaping" are discussed here. Although its principle is so simple, the photo-shaping is a system where high technologies are combined. Many kinds of research resources such as computer science, chemistry, mechanics and laser optics were introduced. Among them the performance of the resin is the most decisive. Imperfectness of the resin has been covered by other technologies for the time being. The users say that the systems have improved much more sinoe they puton sale first.

The requirements for the resin are diverse by their application fields;

(1)Low distortion and shrinkage for the precise model,

(2)Heat resistance for the dinect shaping of the injection mold,

(3)High photosensitivity for the economical system,

(4)Tenacity for the functional checking model,

(5)Completeness of burn out for the investment casting.

The key of advancing these methods technically and commercially is the improvement of the resin and RP industries are waiting for it.

\section{References}

[1]Marutani et al:Photo-shaping, Nikkan Kogyo (19 90).

[2]T.shimizu;Optronics(1996), No.4, pp139-144.

[3]Kuzusako,Harimaya;Proc. 10th Rapid prototyping symposium(May 1996,Kyoto),pp1-5

[4]Catalog of Cubital Co.

[5] S.Okada,MImade, H.Miyauchi, H.Goto:Proc. 11th Rapid prototyping symposium(Nov. 1996, Saitama), pp59-61.

[6] T.Himer,T.Nakagawa,H.Noguchi:Proc. 10th Rapid prototyping symposium(May 1996, Kyoto), pp31-36.

[7]A.Kazama,A.Kamimura,S.Murakami,H.Nakaj̈m aidib, pp11-15.

[8] Y.Marutani, T.Kamitani:Proc. 7Th International Conf. Rapid prototyping( April 1997,Sanfrancisoo) (to be published).

[9]Y.Marutani, K.Kamitani:Proc. Solid freeform Fabrication 96 (Aug. 1996, Austin) pp321-326.

[10]Y.Marutani,T.Nakai:Review of laser Engineerng,22(1995),pp985-992.

[11]Tamura:Die and Mold 11(1996),pp331-337. [12]Ishikawa: Proc. 4th Rapid prototyping symposium (May 1994, Nagoya),pp37-41.

[13]T.Takagi,H.Nakajima: Proc. 3rd Rapid prototyping symposium (Nov.1992Tokyo), pp37-41. 